\title{
Analysis of online questions about dental hygienist: Case report in Naver Intelligent Network
}

\author{
Sang-Hwan Oh, Na-Ri Shin, and Soo-Jeong Hwang* \\ Department of Dental Hygiene, College of Medical Science, Konyang University, Daejeon 35365, Republic of Korea
}

(Received Nov 6, 2017; Revised version received [1] Jan 4, 2018 [2] Feb 3, 2018; Accepted Feb 27, 2018)

\begin{abstract}
Current trends note that individuals utilize the information provided through online knowledge search services, to solve their curiosity for news and world events, as well as to read information written by others and acquire new knowledge on a variety of subjects. The purpose of this study was to analyze the questions about dental hygienists, in an effort to know which information they would want to gain through a web-based question and answer service. The questions were available through the service from October 2002 to March 2017, and it was through the web-based question-answer service in Naver that is called knowledge iN which was searched, using the key words of chigwawiseangsa and chiwiseangsa in the Korean language. As a result, there were a total of 1693 questions about the profession of the dental hygienist occupation which were extracted and analyzed. In particular, it was noted that the high school students asked about the occupation of dental hygienists the most. The middle and high school students required the information about the required entrance examination and employment in this growing field of study, and it was noted that the university students and dental hygienists wanted to know more information regarding statistics of available employment of dental hygienists (such the job options that would be available as upon graduation from their certificate program, etc.). The information orders that the questioners wanted answered were determined and identified as follows: employment (39.3\%), entrance examination (18.8\%), working conditions (11.5\%), tasks $(11.3 \%)$, and curriculum (9.4\%). When the information captured from the questions from the participants and questioners were assorted in detail, the detail information orders that the questioners wanted were as follows: information on applicable places of study, such as the available university $(13.0 \%)$, annual salary $(11.5 \%)$, prospects $(10.3 \%)$. overseas employment $(9.3 \%)$, and public dental hygienists $(8.7 \%)$.
\end{abstract}

KEY WORDS: Dental hygienist, Job, Online

\section{서 론}

정보통신기술의 발달로 소비자의 정보 요구 및 이에 대 한 충족이 인터넷을 기반으로 하는 경우가 점점 많아지고 있다[1]. 지식검색이란 네티즌끼리 묻고 답하며 정보를 만 들어 가는 커뮤니티서비스로서 누군가 질문을 올리면 자 신이 아는 범위 내에서 답변을 해 일종의 온라인 백과사 전을 만들어가는 형식으로 이루어진다. 지식검색은 네티 즌이 자발적으로 참여해 질문과 답변을 한다는 점에서 관 련 사이트 안내 수준에 그치던 기존의 검색서비스와 차별 화된 쌍방향 검색이다. 이제 사람들은 수많은 궁금증에 대

\footnotetext{
*Corresponding author: Soo-Jeong Hwang

Department of Dental Hygiene, College of Medical Science, Konyang University, 158 Gwanjeodong-ro, Seo-gu, Daejeon 35365, Republic of Korea Tel.: +82-42-600-6381, Fax: +82-42-600-6565

E-mail: denthwang@konyang.ac.kr
}

해 지식검색서비스를 이용하여 궁금증을 해소하고 지식을 습득한다[2].

2000년 10월 인터넷 한겨레에서 만든 국내 지식검색서 비스의 최초 모델인 디비딕을 시작으로 2002년 10월 네이 버가 선보인 지식iN이 선풍적 인기를 끌면서 네티즌들의 참여가 본격화 되었다[3]. 1990년대 후반부터 IT열풍을 타 고 인터넷 포털 사업자들이 경쟁적으로 검색서비스를 제 공해 온 가운데, NHN 네이버의 경우 2011년 기준 국내 검색 서비스 시장에서 $60 \sim 70 \%$ 의 시장을 점유하였고[4], 2016년 $48 \%$ 로 감소하기는 하였으나(http://www.pitchone.co.kr/7235/) 국내 검색시장의 지배적사업자로 여전히 위치하고 있는 상황이다. 현재 우리나라에서는 네이버의 지식iN을 통하 여 자기 자신이 가지고 있는 전문지식에서 생활상식까지 손쉽게 교환하거나 공유할 수 있다[5].

치과를 방문하기 전에 인터넷을 통해 정보를 습득하는 사람이 $33.6 \%$ 이고[6] 치위생학과 입학 지원 시 포털사이 트 검색을 하는 사람이 $55.9 \%$ 에 이른다[7], 온라인 정보를 
이용한 국내연구 관련 검색결과, 네이버, 네이트, 다음에 등록된 지식서비스를 이용하여 구강관리에 대한 인터넷 상담 4,212건의 질문을 분석한 연구가 존재하였다[8]. 지 식검색서비스를 이용하지는 않았지만 17 대 대선과 18 대 총선 기간 중 뉴스와 신문의 보건의료정책 선거 보도 기 사에 관한 분석연구[9]가 있었다. 그럼에도 불구하고, 타 분야에 비해 치과분야에 있어서 온라인 정보를 이용한 연 구는 활발하지 않아 보인다.

치과위생사가 환자들에게 간호사나 언니, 아가씨 등으로 불리는 경우가 $30 \%$ 가 넘는 것을 고려할 때[10] 일반인들의 치과위생사에 대한 인식은 부족하다. 이에 따라 대한치과위 생사협회는 치과위생사를 대중들에게 알리기 위해서 2017년 도에 지하철, 버스 등 외부광고를 포함한 대중광고를 집행하 였다 (http://news.kdha.or.kr/news/articleView.html?idxno=7164). 본 연구는 치과위생사에 대한 정확한 인식을 위해서는 대 중들이 치과위생사에 대한 어떠한 정보를 알기 원하는지 조사할 필요가 있다고 판단되었다. 따라서 국내에서 검색 시장 1 위인 네이버의 지식iN에서 법적명칭인 치과위생사 와 통용명칭으로 불리는 치위생사에 대한 질문을 검색하 여, 일반인을 대상으로 치과위생사 직군에 대한 정보 요구 를 분석하고자 한다.

\section{검색보고}

Fig. 1과 같이, 양방향 지식검색서비스인 네이버의 지식

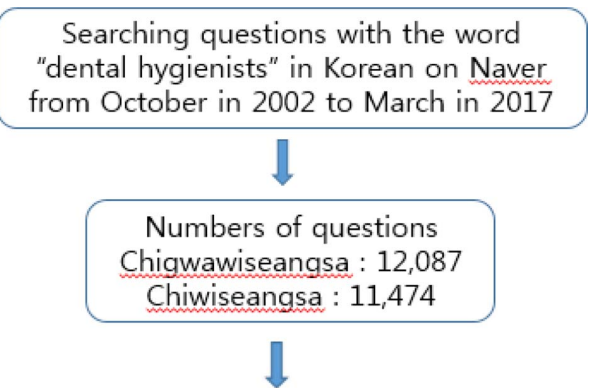

Selecting questions with the topic about "dental hygienists" among them

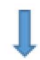

Final questions

Chigwawiseangsa : 798

Chiwiseangsa : 895

Fig. 1. The flow chart of this study.

$\mathrm{iN}$ 에서 치과위생사와 치위생사라는 키워드를 이용하여 2002년 10월부터 2017년 3월 사이에 올라온 질문으로 검 색하였다. 치과위생사로 검색한 결과는 총 12,087 개, 치위 생사로 검색한 결과는 총 11,474 개 이었다. 이중 치과치료 나 구강보건과 관련된 질문을 제외하고 치과위생사 직군 에 관한 질문만 추출하였다. 그 결과 치과위생사로 검색했 을 때 총 798 개의 질문과 치위생사로 검색했을 때 895 개 의 질문, 총 1,693 개 질문이 자료로 추출되었다. 포털사이

Table 1. The frequencies of request informations according to the questioner groups when "Chigwawiseangsa" and "Chiwiseangsa" in Korean were searched through Naver Jisik iN data from October in 2002 to March in 2017

\begin{tabular}{llrrrrr}
\hline \multirow{2}{*}{ Category } & \multicolumn{1}{c}{ Keywords } & $\begin{array}{c}\text { Middle school } \\
\text { students }\end{array}$ & $\begin{array}{c}\text { High school } \\
\text { students }\end{array}$ & $\begin{array}{c}\text { College } \\
\text { students }\end{array}$ & $\begin{array}{c}\text { Dental } \\
\text { Hygienist }\end{array}$ & Etc.* $^{*}$ \\
\hline \multirow{2}{*}{ Total } & Chigwawiseangsa & $111(13.9)$ & $300(37.6)$ & $122(15.3)$ & $157(19.7)$ & $108(13.5)$ \\
& Chiwiseangsa & $108(12.1)$ & $373(41.7)$ & $141(15.8)$ & $119(13.3)$ & $154(17.2)$ \\
Entrance examination & Chigwawiseangsa & $57(29.7)$ & $122(63.5)$ & $5(2.6)$ & $3(1.5)$ & $5(2.6)$ \\
& Chiwiseangsa & $32(25.2)$ & $78(61.4)$ & $9(7.1)$ & $2(1.6)$ & $6(4.7)$ \\
Employment & Chigwawiseangsa & $25(7.9)$ & $103(32.6)$ & $64(20.3)$ & $109(34.5)$ & $15(4.7)$ \\
& Chiwiseangsa & $29(8.3)$ & $132(37.8)$ & $60(17.2)$ & $87(24.9)$ & $41(11.7)$ \\
Working conditions & Chigwawiseangsa & $14(13.7)$ & $35(34.3)$ & $17(16.7)$ & $21(20.6)$ & $15(14.7)$ \\
& Chiwiseangsa & $14(11.0)$ & $67(52.8)$ & $21(16.5)$ & $11(8.7)$ & $13(10.2)$ \\
Curriculum & Chigwawiseangsa & $3(6.3)$ & $18(37.5)$ & $17(35.4)$ & $1(2.0)$ & $9(18.8)$ \\
& Chiwiseangsa & $24(21.6)$ & $50(45.0)$ & $25(22.5)$ & $0(0.0)$ & $12(10.8)$ \\
Task & Chigwawiseangsa & $9(9.5)$ & $21(22.3)$ & $10(10.6)$ & $1(1.0)$ & $53(56.4)$ \\
& Chiwiseangsa & $6(6.2)$ & $21(21.6)$ & $8(8.2)$ & $2(2.1)$ & $60(61.9)$ \\
Etc & Chigwawiseangsa & $3(7.5)$ & $1(2.5)$ & $9(22.5)$ & $22(55.0)$ & $11(27.5)$ \\
& Chiwiseangsa & $2(2.4)$ & $25(29.8)$ & $18(21.4)$ & $17(20.2)$ & $22(26.2)$ \\
\hline
\end{tabular}

"Chigwawiseangsa" is a legal term for a dental hygienist in Korea.

"Chiwiseangsa" is not a legal term for a dental hygienist in Korea, but is widely used by the general public.

"means the questioners that didn't show information about themselves. 
Table 2. The frequencies of details of request informations when "chigwawiseangsa" and "chiwiseangsa" in Korean were searched through Naver Jisik iN data from October in 2002 to March in 2017

\begin{tabular}{|c|c|c|c|}
\hline Category & $\begin{array}{c}\text { Chigwawiseangsa } \\
\mathrm{N}(\%)\end{array}$ & $\begin{array}{c}\text { Chiwiseangsa } \\
\mathrm{N}(\%)\end{array}$ & $\begin{array}{l}\text { Total } \\
\mathrm{N}(\%)\end{array}$ \\
\hline Entrance examination & $192(24.1)$ & $127(14.2)$ & $319(18.8)$ \\
\hline University & $121(15.2)$ & $99(11.1)$ & $220(13.0)$ \\
\hline Admission grade & 71(8.9) & $28(3.1)$ & $99(5.8)$ \\
\hline Employment & $316(39.6)$ & $349(35.4)$ & $665(39.3)$ \\
\hline Public dental hygienists & $77(9.6)$ & $70(7.8)$ & $147(8.7)$ \\
\hline Prospect & $80(10.0)$ & $95(10.6)$ & $175(10.3)$ \\
\hline Certificate & $48(6.0)$ & $40(4.5)$ & $88(5.2)$ \\
\hline Expectancy of career & $36(4.6)$ & $62(6.9)$ & $98(5.8)$ \\
\hline Overseas employment & $75(9.4)$ & $82(9.2)$ & $157(9.3)$ \\
\hline Working conditions & $102(12.8)$ & $127(14.2)$ & $229(13.5)$ \\
\hline Annual salary & $84(10.5)$ & $110(12.3)$ & 194(11.5) \\
\hline Welfare & $13(1.6)$ & $14(1.6)$ & $27(1.6)$ \\
\hline Duty hours & $5(0.6)$ & $3(0.3)$ & $8(0.4)$ \\
\hline Curriculum & $48(6.0)$ & $111(12.4)$ & $159(9.4)$ \\
\hline Licensing examination & $29(3.6)$ & $40(4.5)$ & $69(4.1)$ \\
\hline Content of education & $19(2.4)$ & 71(7.9) & $90(5.4)$ \\
\hline Task & $94(11.8)$ & $97(10.8)$ & 191(11.3) \\
\hline Etc & $40(5.0)$ & $84(9.4)$ & $124(7.3)$ \\
\hline Male & $6(0.7)$ & $22(2.5)$ & $28(1.7)$ \\
\hline Total & $798(100.0)$ & $895(100.0)$ & $1693(100.0)$ \\
\hline
\end{tabular}

"Chigwawiseangsa" is a legal term for a dental hygienist in Korea.

"Chiwiseangsa" is not a legal term for a dental hygienist in Korea, but is widely used by the general public.

트의 지식검색기능을 가장 많이 이용하는 것은 10-30대라 는 선행연구[6]가 있었고 본 조사에서도 10-20대가 대다수 를 차지하는 것으로 판단되어 질문 대상자의 특성을 연령 대별로 중학생, 고등학생, 대학생, 기타(질문자의 특성에 대한 정보가 없음)로 구분한 후 질문 유형을 분석하였다. 질문자가 치과위생사임을 밝힌 질문이 276개(16.3\%)였으 며, 치과위생사는 질문유형이 일반인과 다를 것으로 예상 되어 대상자 분류를 따로 하는 것이 적절할 것이라고 판 단되어 별도로 분류하였다. 각 질문의 주제별 분류는 연구 자들의 논의를 통해 합의되는 카테고리로 지정하였으며, 입시, 취업, 근무조건, 교육과정, 업무, 기타로 분류하였다. 질문대상자의 특성에 따라 정보요구도의 빈도를 분석하 였다(Table 1). 치과위생사로 검색 시에는 고등학생, 치과 위생사, 대학생, 중학생, 기타 순이었고 치위생사로 검색 시에는 고등학생, 기타, 대학생, 치과위생사, 중학생 순이 었다. 대상자에 따른 질문유형을 살펴보면 중학생과 고등 학생은 입시관련 질문이 가장 많았고 대학생과 치과위생 사는 취업관련 질문이 가장 많았다. 기타군에서는 치과위 생사의 업무에 관한 질문이 가장 많았다.

본 연구에 수집된 1,693 개의 질문유형을 확인하면서 연 구자들의 합의에 따라 하위 카테고리로 분류하였다 (Table 2). 입시는 대학정보, 입학성적으로 분류하고, 취업
은 공무원, 전망, 자격증, 직업수명, 해외취업으로 분류하 였다. 근무조건은 연봉, 복지, 근무시간으로 분류하였고 교 육과정은 국가고시, 교육내용으로 분류하였다. 업무의 경 우 다양한 질문으로 이루어져 있어서 하위 카테고리를 지 정하지 않았으며, 기타의 경우 남자치과위생사에 관한 질 문이 하위 카레고리로 지정하였고, 그 외의 다양한 질문으 로 구성되었다.

Table 2 와 같이 치과위생사와 치위생사로 각각 검색 시, 질문의 비율은 치과위생사에서는 입시, 취업 쪽의 비율이 높았고, 치위생사에서는 근무조건, 교육과정, 기타 쪽의 비 율이 높았다. 전체 질문 중 가장 많은 것은 취업으로 665 건을 차지하였고 그 중에서 전망, 해외취업, 공무원 비율 의 순이었다. 그 다음으로는 입시로 319 건이었고, 그 중 대학정보에 관한 질문이 가장 많았다. 세 번째는 근무조건 으로 229건이었으며, 그 중 연봉에 관한 질문이 많았다. 네 번째로는 업무에 관한 질문이 191건이었다. 기타 중에 서는 남자치과위생사에 대한 질문이 28 건이었다.

\section{고 찰}

급변하는 지식 환경 속에서 온라인을 통한 지식협업의 
성장은 두드러지고 있다. 인터넷 환경의 집단지성을 대표 하는 위키피디아를 비롯해서 한국의 협업형 집단지성인 지식iN, 독립되고 분산된 정보를 고도로 발달된 기술과 알 고리즘을 통하여 새로운 지식을 만들어 내는 페이지랭크 (PageRank)까지 통합형 집단지성은 종이 지식을 넘어서 다양하게 발달하고 있다[11]. 집단지성에 의한 온라인 지 식환경은 개방성, 평등성, 다원성과 같은 특성을 지니고 있고 서로 다른 견해가 있음을 공평하게 반영하는 것이 그 장점이다[12]. 온라인 지식공유에 있어서 참여자의 특성분 석[13], 질문분석 $[8,14,15]$, 검색행태 분석 [1,16], 특정용어 사용 분석[17] 등 다양한 연구들이 2000년대 들어서 시행 되었다.

본 연구에서는 네이버 지식iN서비스를 검색하여 치과위 생사, 치위생사라는 용어를 키워드로 사용하여 치과위생 사 직군에 대한의 질문 총 1,693 개의 질문 유형을 분석했 다. 치과위생사로 검색을 하는 비율이 $47.1 \%$, 치위생사로 검색을 하는 비율이 $52.9 \%$ 로 약간 더 높게 나타났으며, 고 등학생, 대학생, 기타에서는 치위생사로 검색을 하는 비율 이 더 높았다. 이에 치과위생사와 치위생사라는 용어가 현 실에서 혼재되고 있음을 보여주는 사실임을 고려해 볼 때 관련 단체에서 직군에 관한 정확한 명칭 홍보가 필요할 것 으로 사료된다.

인터넷 검색을 통해 정보를 얻는 연령대가 10-30대라고 연구된 선행연구[6]와 같이, 질문자의 중학생 비율이 $12.9 \%$ (219건), 고등학생 비율이 $39.8 \%(673$ 건), 대학생 비율 이 $15.5 \%$ (263건)로 $10-20$ 대의 비율이 $68.2 \%$ (1155건)에 달 하고 있다. 본 연구목적이 일반인의 치과위생사 직군에 대 한 정보 요구를 분석하고자 함임을 볼 때 특정 연령대의 대 상자에 치우친 정보를 분석한 것이므로 이후 연령대에 관 한 조사를 위해서는 인터넷 검색이 아닌 다른 연구방법을 고려해야 할 것이다. 연령대가 10-20대로 치우쳐 있어 분석 질문의 전체적 비율도 취업과 입시 쪽에서 높게 나타난 것 으로 보인다. 취업에 있어서 치과위생사의 취업전망이나 해외취업, 공무원취업, 직업수명 순으로 빈도가 나타난 것 은 최근 심각한 청년실업율의 증가와 일자리의 질, 직업 안 정성 등이 문제가 되었기 때문[18]으로 사료된다.

결과 중 특이한 사항은 질문자의 특성을 추정할 수 없 는 기타로 분류된 질문자들은 262 건의 질문 중에서 $43.1 \%(113$ 건)가 업무에 관한 질문이라는 것이다. 치과위생 사가 치과 내에서 어떠한 행위를 해도 되는지에 관한 질 문이 많았으며, 이는 일반인들은 치과위생사의 법적 업무 를 잘 알지 못하기 때문인 것으로 보인다. 또한, 치과위생 사 관련 단체는 치과위생사의 법적 업무범위가 치석 등 침 착물제거, 불소도포, 임시충전, 임시부착물 장착 및 제거, 인상채득, 교정용 호선의 장착 및 제거, 그 밖의 치아 및 구강질환의 예방과 위생 등 폐쇄식으로 되어 있는 문제점
과 법으로 기술되어 있는 업무도 보건복지부의 유권해석 에 의존하는 것에 대해 개선을 할 필요가 있다. 이로 인해 현장에서 치과위생사들의 업무가 법적 업무인지 아닌지에 대한 논란이 되는 경우가 많다[19].

선행연구 $[8,9]$ 는 인터넷 검색 후 수작업으로 모든 질문 을 분류 분석하였고, 본 연구 또한 연구자들에 의한 수작 업으로 모든 질문을 분류 분석하였다. 지식검색서비스가 정착되었음에도 불구하고 답변은 $90 \%$ 이상의 적합성을 보이나 질문의 제목과 전체 질문이 부적합한 지식의 질문 분포가 $56.3 \%$ 를 나타내고 있다는 연구[15]는 여전히 연구 자가 관련 정보를 분석하기 위해서 질문 내용을 읽고 해 석하는 수작업이 필요할 수도 있다는 것을 방증하는 것이 기도 하다. 그러나 온라인상에 존재하는 자료의 양이 갈수 록 방대하며 더 많은 정보가 업데이트되고 있는 현실에서 연구자가 수작업으로 분류하는 것은 연구자로 하여금 상 당한 시간을 요하게 된다. 따라서, 온라인에서의 정보를 이용하고 수작업이 아닌 컴퓨터에 의한 분석이 이루어질 수 있도록 구강보건분야에서도 빅데이터를 연구할 수 있 는 연구자의 필요성이 앞으로 더 강조되리라고 사료된다.

의사, 치과의사, 한의사는 온라인 전문상담원으로서 수 백에서 수천 명의 인원이 확보되어 있고[14,17] 관련 전문 가들이 정확한 지식전달을 위해 활동하고 있지만, 치과위 생사와 관련된 답변의 경우 응답자의 특성이 불분명한 경 우가 많아 응답자 특성에 관한 사항은 조사에서 배제하였 다. 정확한 연구결과를 위해서 지식검색사이트는 질문자 와 답변자의 연령이나 학력, 직업 등 정보를 알 수 있도록 개선된다면 신뢰성은 확보가 되겠지만, 개인 정보유출과 같은 문제와 결부되어서 어려울 수 있다. 따라서, 치과위 생사와 관련된 지식검색서비스의 질문에 대해 치과위생사 단체들이 앞장서서 정확한 정보를 전달하도록 제안하는 바이다. 또한 본 연구에서는 국내에서 가장 많이 사용하는 네이버의 검색엔진만을 선정하였으나, 그 외에 다양한 지 식검색서비스가 있기 때문에 이후 다양한 검색사이트를 추가하여 연구하는 것이 필요하다.

\section{Conflict of Interest}

The authors declare that they have no competing interests.

\section{ORCID}

$\begin{array}{ll}\text { Sang-Hwan Oh } & 0000-0002-5944-0129 \\ \text { Na-Ri Shin } & 0000-0003-4417-4638 \\ \text { Soo-Jeong Hwang } & 0000-0003-4725-1512\end{array}$




\section{References}

1. Park SY, Lee JH, Kim JS. An analysis of query types and topics submitted to Naver JKLISS 2005;39:265-278. doi: 10.4275/KSLIS.2005.39.1.265.

2. Kim HY. Study of knowledge and knowledge search in information society: case study of Naver knowledge service. Information \& communications policy 2006;18:119.

3. Park JB, Jeong DY. An empirical study on web-based question-answer services. JKOSIM 2004;21:83-98. doi: 10.3743/KOSIM.2004.21.3.083.

4. Gawk GT. Direction of improvement in Naver search service. Review of Korean Society for Internet Information 2011;12:37-44.

5. Han JW, Yoo CW, Choi YC. Factor influencing the internet knowledge sharing. Journal of agricultural extension \& community development 2009:16:153-180.

6. Choi HS. Impact of information search on the choice of dental institution. J Dent Hyg Sci 2009;9:587-592.

7. Hwang SJ, Koong HS, Kang KH, Oh SH. The analysis of college entrance tendency in applicants to dental hygiene department. J Korean Soc Dent Hyg 2014;14:915. doi: 10.13065/jksdh.2014.14.01.9.

8. Kim MJ, Yang HJ. Research on internet counselling for oral health, KJOHSM 2013;7:251-260. doi: 10.12811/ kshsm.2013.7.3.251.

9. Choi JA, Yang MJ, Chung WG, Kim CS. Survey and analysis of major newspapers and broadcastings about public health policy: through 17 th presidential election in 2007 and 18th election in 2008. J Dent Hyg Sci 2009: 9:69-74.
10. Park SY, Won YS. Recognition of health care workers for dental hygienists. KJOHSM 2012:6:127-140. doi: 10.12811/kshsm.2012.6.3.127.

11. Yoon WC, Yi MY. A new e-learning system based on integrative collective intelligence. Telecommunications Review 2010;20:943-952.

12. Lee HE. Techno-cultural meanings of the information in Wikipedia: collective intelligence and knowledge power. JOCS 2009;9:461-497.

13. Park HJ, Lee HJ, Kim JW. Participation level in online knowledge sharing: behavioral approach on Wikipedia, J Intell Inform Syst 2013;19:97-121. doi: 10.13088/jiis. 2013.19.4.097.

14. Kim YK, Lim BM. Internet health counseling for korean medicine in the Naver Jisik-iN. SPKOM 2013;17:51-63.

15. Park SY, Lee JH, Jeon JW. Evaluation of the documents from the Web-based Question and Answer Service, JKLISS 2006;40:299-314. doi: 10.4275/KSLIS.2006.40. 2.299 .

16. Lee JH, Park SY, Kwon HS. Information seeking behavior of the Naver users via query log analysis. JKOSIM 2003;20:27-41. doi: 10.3743/KOSIM.2003.20.2.027.

17. Kim SM, Lee SW. Mun SJ. Analysis of the mibyeong concept and user on the internet.-Focusing on Naver Jisik-iN Q\&A, Cafépost-. SPKOM 2017;21:95-106.

18. Preut H. European and South Korean unemployed youth in times of crisis: An analysis of youth unemployment related problems and policies for the young. NEA 2016:31:205-234.

19. Hwang SJ, Koong HS, Lee SH. Difference of perception of the duties of dental hygienist between dentists and dental hygiene students in an area. JKADA 2017:5:1-12. 